Vol. 45 No. 2/1998

$587-539$

QUARTERLY

\title{
Communication
}

\section{Template-directed base pairing of 2-chloro-2'-deoxyadenosine catalyzed by AMV reverse transcriptase ${ }^{\star \otimes}$}

\author{
Agnieszka M. Bukowska-Maciejewska and Jarosław T. Kuśmierek ${ }^{\circledR}$ \\ Department of Molecular Biology, Institute of Biochemistry and Biophysics, Polish Academy of \\ Sciences, Warsaw, Poland
}

Key words: 2-chloro-2-deoxyadenosine, base pairing, AMV reverse transcriptase

\begin{abstract}
2-Chloro-2 ${ }^{\prime}$-deoxyadenine (2CldA) is used for treatment of several lymphoid malignancies. Since this drug is incorporated into DNA, we have undertaken studies on base pairing of 2-chloroadenine (2CLA). 2CldA phosphoramidite was synthesized and used for preparation of 25-mer templates with 2 ClA located at site 21 from the 3 'end. Kinetic parameters $\left(K_{m}\right.$ and $\left.V_{\max }\right)$ for the incorporation of deoxynucleoside-5'triphosphates by AMV reverse transcriptase opposite the 2CLA template, as well as for the extension of 2ClA $\cdot T$ pair, were determined. The efficiency $\left(V_{\max } / K_{m}\right)$ of incorporation of dGTP, dCTP, and dATP opposite 2ClA is at least one order of magnitude lower than opposite unmodified A. The efficiency of incorporation of dTTP opposite 2CLA is about 30-fold lower than opposite $\mathrm{A}$ and extension of $2 \mathrm{ClA} \cdot \mathrm{T}$ pair is 3-fold lower than of A T T pair. From the analysis of the parameters of dTTP incorporation we conclude that formation of 2ClA $\cdot \mathrm{T}$ pair is thermodynamically, but not kinetically controlled. The difference in binding energy $(\Delta \Delta G)$ between $2 \mathrm{ClA} \cdot T$ and $A \cdot T$ pairs in the environment of the polymerase active site is $2 \mathrm{kcal} / \mathrm{mol}$. Our results indicate that the presence of 2ClA in DNA slows down replication, but does not lead to basesubstitution mutations.
\end{abstract}

2-Chloro-2' -deoxyadenosine (2CldA) is markedly toxic to many leukemia and lymphoid cell specimens as well as to monocytes. It is used for treatment of several lymphoid malignancies, such as chronic lymphocytic leukemia, nonHodgkin's lymphoma, cutaneous T-cell

*Paper presented at the Conference on "Mechanisms of DNA Repair and Mutagenesis" Commemorating the $100^{\text {th }}$ Anniversary of the Discovery of Polonium and Radium, October, 1997, Warsaw, Poland.

'Supported by the State Committee for Scientific Research (KBN 6 P203 01906).

${ }^{\Xi}$ Corresponding author: J.T. Kuśmierek, Institute of Biochemistry and Biophysics, Polish Academy of Sciences, A. Pawińskiego 5A, 02-106 Warszawa, Poland; e-mail: jareq@ibbrain.ibb.waw.pl

Abbreviations: AMV RT, Avian Myeloblastosis Virus reverse transcriptase; 2ClA, 2-chloroadenine or 2chloroadenine residue in DNA; 2CldA, 2-chloro-2'-deoxyadenosine; dNTP, deoxynucleoside-5'. triphosphate. 
lymphomas, chronic granulocytic leukemia and others. The best and most durable effects have been seen with hairy cell leukemia.

In cell cultures $2 \mathrm{Cl}$ di is readily taken up by a specific nucleoside transport system and is converted to the active triphosphate form (2CldATP) by cellular kinases. The substitution of chlorine for hydrogen at position 2 of purine ring renders the nucleoside resistant to cellular adenosine deaminase, which results in rapid accumulation of its mono-, diand triphosphorylated forms in the cell. The mechanism of action, pharmacological features of and clinical trial results on $2 \mathrm{CldA}$ were reviewed recently by Saven \& Piro [1].

Since 2CldA is incorporated into DNA in cells, it can alter its template properties during replication. The coding properties of $2 \mathrm{ClA}$ may differ from those of unmodified adenine and, moreover, the presence of 2ClA in DNA template can hinder the replication process. The aim of our study was to answer the question whether 2ClA present in a template can lead to base-substitution mutation and how it influences the rate of replication.

\section{MATERIALS AND METHODS}

The preparation of $5^{\prime}-O(4,4$-dimethoxytriphenylmethyl)-3' $-O$-[(diisopropylamino(2cyanoethoxy)phosphino]-2-chloro- $N^{6}$-benzoyl2 '-deoxyadenosine was accomplished following procedures elaborated for 2 '-deoxyadenosine and its adducts $[2,3]$. The fully protected phosphoramidite of $2 \mathrm{CldA}$ was used for preparation of oligonucleotides with the aid of Gene
Assembler+ (Pharmacia LKB) synthesizer. Two 25-mer templates with 2ClA at the position 21 from the 3 '-termini (M11, M21), two 25-mers with unmodified $A$ at the same position (M1, M2) and two complementary 20-mer primers $(\mathrm{P} 1, \mathrm{P} 2)$ were prepared. The sequence of all templates and primers is fixed (shown for the P1 M11 duplex, Fig.1) with the exception of the positions 20,21 and 22 in templates and 3 '-terminal position in primers. The variable bases in all templates and primers are shown in Table 1. All prepared oligonucleotides were 5 '-labelled and then subjected to polyacrylamide gel electrophoresis (PAGE). Since their electrophoretic mobility corresponded to the expected length, and the amount of abortive products did not exceed $10-20 \%$, they were used for experiments without any further purification. The replication of templates in the presence of various sets of dNTPs gave the expected patterns after PAGE (not shown), which indicated that 2ClA was present in templates at the chosen 21 position and that the 5 '-termini sequences of templates were consistent with expectation. Additionally, the presence of $2 \mathrm{CldA}$ in templates was confirmed by HPLC analysis after enzymatic digestion to deoxynucleosides. Kinetic parameters for the site-specific incorporation of dNTPs by AMV RT were determined using a methodology elaborated in Goodman's laboratory $[4,5]$. Primers were 5'-labelled with T4 polynucleotide kinase and $\left[\gamma^{32} \mathrm{P}\right] \mathrm{ATP}$ usually $50 \mu \mathrm{Ci}$ per $0.0007 \mathrm{~A}_{260}$ of primer was used and annealed to complementary templates. The AMV RT reaction mixtures contained in 6 $\mu \mathrm{L}: 50 \mathrm{mM}$ Tris/HCl (pH 7.8), $3 \mathrm{mM} \mathrm{MgCl}_{2}, 1$

\section{3'- CCGCCCATGgCtCGagCtTA P1 5'-CCGTXTGCGGGTACCGAGCTCGAAT M11}

Figure 1. The sequence of M11 template with annealed complementary P1 primer (X - 2ClA).

The other oligonucleotides used in the experiments had an identical sequence with the exception of positions 20,21 and 22 in templates and $3^{\prime}$-terminal position in primer. The variable parts of the sequence in templates and primers are shown in Table 1. 
mM DTT, $0.005 \mathrm{~A}_{260} / \mathrm{mL}$ of ${ }^{32}$ P-labelled primer and $0.0075 \mathrm{~A}_{260} / \mathrm{mL}$ of template (about 1.2 fold molar excess of template over primer), dNTPs appropriate for an experiment at adequate concentrations and 0.1 or 0.3 units of AMV RT. The higher amount of enzyme was used in misincorporation experi- same sequences. The results indicate that $2 \mathrm{ClA}$ is a poorer counterpart of $\mathrm{T}$ than unmodified A but is still capable of forming a base pair with $\mathrm{T}$. The influence of neighbouring bases in template on kinetic parameters is also observed but this effect was similar for both bases studied.

Table 1. Kinetic constants of dTTP incorporation opposite $\mathrm{A}$ or $2 \mathrm{ClA}$ determined in two different sequences

\begin{tabular}{|c|c|c|c|c|c|}
\hline Primer/template $e^{(a)}$ & $\begin{array}{l}K_{\mathrm{m}} \\
(\mu \mathrm{M})\end{array}$ & $V_{\max }\left(\mathrm{I}_{2} / \mathrm{I}_{1}\right)^{(\mathrm{b})}$ & $\begin{array}{l}V_{\max } / K_{m} \\
\left(\mu \mathrm{M}^{-1}\right)^{(\mathrm{c})}\end{array}$ & $\mathrm{f}_{\mathrm{rel}}{ }^{(d)}$ & $\begin{array}{l}\Delta \Delta \mathrm{G}^{(\mathrm{e})} \\
\left(\mathrm{kcal} \cdot \mathrm{mol}^{-1}\right)\end{array}$ \\
\hline $\begin{array}{ll}\text { P1 } & \ldots \text { A } \\
\text { M1 } & \ldots \text { TAT... }\end{array}$ & $0.08 \pm 0.04$ & $0.26 \pm 0.11$ & 3.25 & 1.00 & \\
\hline $\begin{array}{l}\text { P1 } \ldots \mathrm{A} \\
\mathrm{M} 11 \ldots \mathrm{TXT} . . .\end{array}$ & $1.82 \pm 1.00$ & $0.22 \pm 0.08$ & 0.12 & 0.04 & 1.9 \\
\hline $\begin{array}{ll}\text { P2 } & \ldots \mathrm{C} \\
\text { M2 } & \ldots \mathrm{GAG} . . .\end{array}$ & $0.10 \pm 0.05$ & $0.28 \pm 0.09$ & 2.80 & 1.00 & \\
\hline $\begin{array}{ll}\mathrm{P} 2 & \ldots \mathrm{C} \\
\mathrm{M} 21 \ldots \mathrm{GXG} . . .\end{array}$ & $3.60 \pm 2.00$ & $0.28 \pm 0.07$ & 0.08 & 0.03 & 2.2 \\
\hline
\end{tabular}

(a) The closest neighborhood of target $A$ or 2 ClA in template and primer is shown (X - 2CLA).

(b) The apparent $K_{\mathrm{m}}$ and $V_{\max }$ values in the Table were calculated from 3-7 independent determinations.

(c) The ratio $V_{\max }$ to $K_{\mathrm{m}}$ represents the incorporation efficiency.

(d) $\mathrm{f}_{\mathrm{rel}}$ - the efficiency of incorporation opposite $2 \mathrm{ClA}$ related to incorporation opposite $\mathrm{A}$ in the same sequence.

(e) The free energy difference for base binding in 2ClA $T$ pair $v s \mathrm{~A} \cdot \mathrm{T}$ pair, calculated from the equation $\Delta \Delta \mathrm{G}=\mathrm{RT} \ln \left[K_{\mathrm{m}}(2 \mathrm{ClA} \cdot \mathrm{T}) / K_{\mathrm{m}}(\mathrm{A} \cdot \mathrm{T})\right]$

ments. In all remaining experiments 0.1 unit of enzyme in $6 \mu \mathrm{L}$ reaction mixtures was used, which corresponds to 1:10 enzyme primer/template ratio.

\section{RESULTS}

Kinetic parameters $\left(K_{\mathrm{m}}\right.$ and $\left.V_{\max }\right)$ for sitespecific incorporation of dNTPs by AMV RT have been measured. The results for dTTP incorporations opposite $\mathrm{A}$ and $2 \mathrm{ClA}$ in two sequences studied are shown in Table 1 . The efficiency of insertion of dTTP opposite 2ClA was 25-35 fold lower than opposite A. Substitution of chlorine at position 2 of adenine strongly influenced the $K_{\mathrm{m}}$ values whereas the $V_{\max }$ values were similar, especially when we compare these values for $\mathrm{A}$ and $2 \mathrm{ClA}$ in the
The extension of $2 \mathrm{ClA} \cdot \mathrm{T}$ pair was studied in P1M11 duplex. The $K_{\mathrm{m}}$ value was higher and $V_{\max }$ was lower in comparison to the values determined for extension of $\mathrm{A} \cdot \mathrm{T}$ pair. The overall effect is manifested by about 3 fold lower efficiency of extension of 2ClA $\cdot \mathrm{T} v s$ A.T pair (Table 2).

The incorporation of dCTP, dGTP and dATP opposite 2ClA and A was also studied (Table 3 ). The $K_{\mathrm{m}}$ values for these reactions were at least three orders of magnitude higher than for incorporation of dTTP. In the case of dATP no measurable incorporation opposite 2CldA was found up to $10 \mathrm{mM}$ dATP. In each case studied the efficiency of insertion of noncomplementary nucleotides opposite $2 \mathrm{CldA}$ was at least one order of magnitude lower than opposite $\mathbf{A}$. 
Table 2. Kinetic constants of extension of $\mathrm{T} \cdot \mathrm{A}$ or $\mathrm{T} \cdot 2 \mathrm{ClA}$ pair ${ }^{(a)}$

\begin{tabular}{|c|c|c|c|c|}
\hline primer/template ${ }^{(b)}$ & $K_{\mathrm{m}}(\mu \mathrm{M})^{(c)}$ & $V_{\max }\left(\mathrm{I}_{2} / \mathrm{I}_{1}\right)^{(\mathrm{c})}$ & $V_{\max } / K_{m}\left(\mu \mathrm{M}^{-1}\right)^{(\mathrm{d})}$ & $f_{\text {rel }}^{(e)}$ \\
\hline $\begin{array}{ll}\mathrm{P} 1 & \ldots \mathrm{AT} \\
\mathrm{M} 1 & \ldots \mathrm{TAT} \ldots\end{array}$ & $0.23 \pm 0.02$ & $0.22 \pm 0.05$ & $x^{2}$ & 1.00 \\
\hline $\begin{array}{l}\text { P1 } \ldots \text { AT } \\
\text { M11 ...TXT... }\end{array}$ & $0.34 \pm 0.26$ & $0.12 \pm 0.05$ & 0.35 & 0.36 \\
\hline
\end{tabular}

${ }^{\text {(a) }}$ Kinetic constants were determined in reactions where 20 -mer P1 primer was elongated by two bases. Reactions were carried on at constant dTTP concentrations ( $1 \mu \mathrm{M}$ and $20 \mu \mathrm{M}$, for M1 and M11, respectively) and at variable dATP concentrations. Under these conditions essentially only disappearance of 20 -mer band and formation of $22-m e r$ band were observed after PAGE and autoradiography.

(b) The closest neighborhood of target $\mathrm{A}$ or 2ClA in template and primer is shown (X -2 CLA).

(c) The apparent $K_{\mathrm{m}}$ and $V_{\max }$ values in the Table were calculated from 4-6 independent determinations.

(d) The ratio $V_{\max }$ to $K_{\mathrm{m}}$ represents the extension efficiency.

${ }^{(e)} f_{\text {rel, }}$, the efficiency of extension of $2 \mathrm{ClA} \cdot \mathrm{T}$ pair related to extension of $\mathrm{A} \cdot \mathrm{T}$ pair.

\section{DISCUSSION}

Previous works have shown that 2CldA can affect DNA synthesis in a twofold manner: (i) 2CldATP slows down DNA replication during its incorporation, (ii) when incorporated into DNA, the template $2 \mathrm{ClA}$ residues hinder the new strand synthesis. Distinct pauses in DNA replication are observed, related to the site of incorporation of 2CldATP opposite template $\mathrm{T}$ or of dTTP opposite template $2 \mathrm{ClA}$, respectively [6-10]. Both cases can be categorised as impaired interaction between 2CIA and $\mathrm{T}$ bases at the polymerase active site, which in-

Table 3. Kinetic constants of noncomplementary dNTP incorporation opposite A or 2ClA

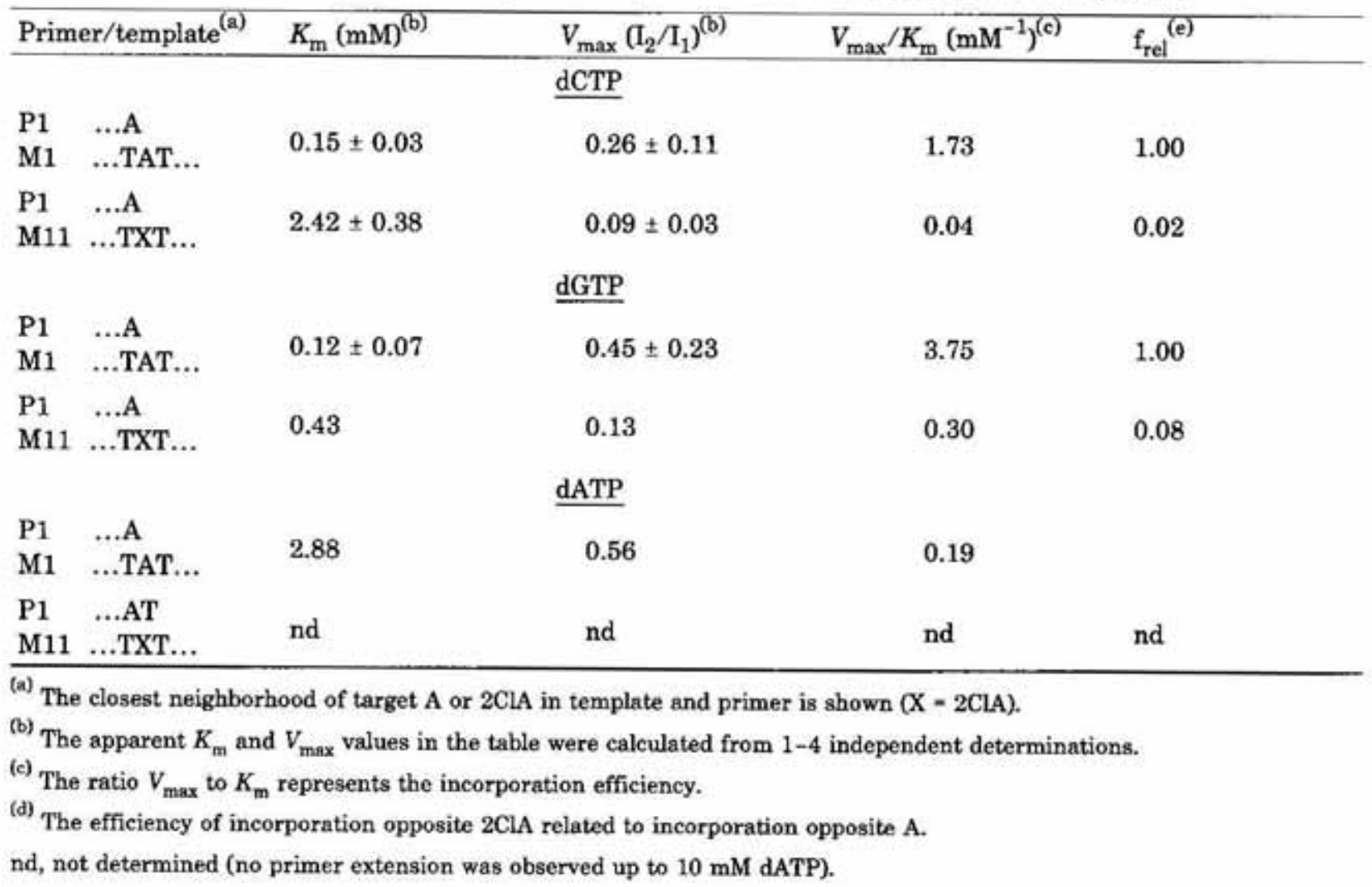


fluences formation of the $2 \mathrm{ClA} \cdot \mathrm{T}$ pair and its extension. In this work we present a quantitative study on interaction of template 2ClA with dTTP (and also other dNTPs) and this is the first report describing determination of kinetic constants for incorporation of dNTPs opposite 2ClA located at a preselected site in oligodeoxynucleotide template, and for extension of the $2 \mathrm{ClA}$ - $\mathrm{T}$ pair. As the replicating enzyme we have used one of the simplest DNA polymerases lacking the proof-reading activity, Avian Myeloblastosis Virus reverse transcriptase (AMV RT).

Our study demonstrated that dTTP is a strongly preferred substrate over other dNTPs in the AMV RT catalysed incorporation opposite template $2 \mathrm{ClA}$. The $K_{\mathrm{m}}$ values for dTTP incorporation are in micromolar range, whereas for incorporation of the remaining dNTPs are in millimolar range (compare results in Table 1 vs Table 3), which indicates that dTTP possesses a relatively high affinity to template 2ClA. This is in accordance with results of other authors, from which it can be concluded that the $2 \mathrm{ClA} \cdot \mathrm{T}$ pair is formed during DNA synthesis, irrespective of whether 2ClA is in the template or it acts as a 5 '-triphosphate [6-10].

We have found that the efficiency of incorporation of dTTP opposite $2 \mathrm{ClA}$ is $25-35$ fold lower than the efficiency opposite A, depending on the template sequence in the vicinity of the target base (Table 1). The discrimination between incorporation of dTTP opposite 2ClA or A is apparently thermodynamically, and rather not kinetically controlled $[5,11]$. The observed decrease in efficiency of dTTP incorporation is parallel to the increase of the $K_{\mathrm{m}}$ values, whereas the $V_{\max }$ values are similar. This means that there is no essential geometrical difference between $2 \mathrm{ClA} \cdot \mathrm{T}$ and $\mathrm{A} \cdot \mathrm{T}$ pairs, and distinction between these pairs is based on the difference in the binding energy $(\Delta \Delta G)$ between them in the environment of the polymerase active site (Table 1).

The experimental results presented here are in good agreement with the results obtained by quantum mechanical (QM) methods (G. Bakalarski \& B. Lesyng, unpublished). The calculated difference in the binding energy between the $2 \mathrm{ClA} \cdot \mathrm{T}$ and $\mathrm{A} \cdot \mathrm{T}$ pairs is 3.4 $\mathrm{kcal} \times \mathrm{mol}^{-1} v s 2 \mathrm{kcal} \times \mathrm{mol}^{-1}$ obtained from the experiments (Table 1). This disparity can be assigned to additional interactions at the polymerase active site which were not taken into account in the QM calculations. The distortion of the geometry of $2 \mathrm{ClA} \cdot \mathrm{T}$ complex caused by substitution of the chlorine atom compared to that one of $\mathrm{A} \cdot \mathrm{T}$ is rather small, which leads to the assumption that the 2ClA-T pair fits quite well the B-DNA structure. The study of the molecular electrostatic potentials revealed $\pi$-electron delocalization in the area of Cl-2 of $2 \mathrm{ClA}$ and $\mathrm{O}-2$ of T. The delocalization of the $\pi$-electrons stabilizes the complex, whereas the classical electrostatic repulsion between $\mathrm{O}-2$ of $\mathrm{T}$ and $\mathrm{Cl}-2$ of $2 \mathrm{ClA}$ destabilizes it. The final effect is the relative destabilization of the whole system by the mentioned above $3.4 \mathrm{kcal} \times \mathrm{mole}^{-1}$ (G. Bakalarski \& B. Lesyng, unpublished).

Our results are in line with the results of Hentosh \& Grippo [10] who studied replication of the DNA fragment in which all deoxyadenosines were replaced by $2 \mathrm{CldA}$. The authors found that bypassing of $2 \mathrm{ClA}$ is rather dNTPs concentration than the reaction time dependent. The about 20 and 50 -fold greater dNTPs concentrations were required for bypassing the $2 \mathrm{ClA}$ sites, compared to the $\mathrm{A}$ sites, by human polymerases $\alpha$ and $\beta$, respectively.

The parameters of $2 \mathrm{ClA} \cdot \mathrm{T}$ pair formation are entirely different from that of natural bases mismatches. The $K_{\mathrm{m}}$ values for dNTP misincorporation by Drosophila polymerase $\alpha$ opposite template $\mathrm{T}$ are over 1000 -fold higher than $K_{\mathrm{m}}$ for incorporation of dATP, corresponding to a difference of 4-5 kcal $\times \mathrm{mol}^{-1}$ in the free energy. Unlike the $2 \mathrm{ClA} \cdot \mathrm{T}$ pair, mismatches are formed with a rate $4-8$ fold lower than the A-T pair, which points to geometrical constrains during the dNTPs misincorporation. The overall efficiency of the mis- 
matches formation is $10^{-5}-10^{-4}$ of that of the $A \cdot T$ pair [12]. The relative efficiency of $2 \mathrm{ClA} \cdot \mathrm{T}$ pair formation is of the order of $10^{-2}$ (Table 1) and is more close to the efficiency of formation of mutagenic mispairs such as $O^{6}$ methylG. $\mathrm{T}$ or $\mathrm{O}^{4}$ methylT. $\mathrm{G}$, which are in the range of $10^{-3}-10^{-2}$, depending on the polymerase used and template sequence at the target site [13].

The extension of the 2ClA $\cdot \mathrm{T}$ pair by AMV RT occurs with about 3-fold lower efficiency than extension of the A-T pair (Table 2). The effect is apparently modest, especially when it is compared with extension of natural mismatches or mutagenic mismatches, where the efficiency of extension is $10^{-2}-10^{-4}$ of that of the normal pair $[12,14,15]$.

Another task of our study was to find whether the presence of 2ClA can lead to basesubstitution mutations. The results in Table 3 show that the frequencies of incorporation of noncomplementary dNTPs opposite 2CldA are at least one order of magnitude lower than opposite A. The lack of miscoding of template 2ClA was also stated in transcription by SP6 RNA polymerase of the DNA fragment in which A residues were replaced by $2 \mathrm{ClA}$ ones [16]. These results indicate that formation of mismatches by template 2ClA is less likely than by unmodified $\mathrm{A}$ and that the presence of 2ClA in DNA will not cause base-substitution mutations.

In conclusion, our studies based on determination of kinetic parameters have shown that the presence of $2 \mathrm{ClA}$ in DNA template diminishes the rate of replication because of lower affinity of incoming dTTP to $2 \mathrm{ClA}$ and lower efficiency of extension of $2 \mathrm{ClA} \cdot \mathrm{T}$ pair, as compared to unmodified A and A-T pair, respectively. These effects are not very large, especially when compared to the effects caused by mismatches. We have shown also that formation of mismatches by 2CIA is less likely than by unmodified $\mathrm{A}$, therefore the presence of 2ClA in DNA will not cause base-substitution mutations.
We are deeply indebted to Dr. Zygmunt Kazimierczuk of the Department of Biophysics, University of Warsaw, for the sample of 2CldA. We also thank Ms. Katarzyna Lichota for HPLC analyses. One of us (A.M.B.M.) thanks the Institute of Biochemistry and Bio physics, PAS, for a predoctoral fellowship.

\section{REF E R E N CES}

1. Saven, A. \& Piro, L.D. (1994) Ann. Intern. Med. 120, 784-791.

2. Fritz, H.J., Frommer, W.B., Kramer, W. \& Werr, W. (1982) in Chemical and Enzymatic Synthesis of Gene Fragments (Gassen, H.G., and Lang, A., eds.) pp. 43-52, Verlag Chemie, Weinheim, Deerfield, Beach, Florida, Basel.

3. Chenna, A. \& Singer, B. (1995) Chem. Res. Toxicol. 8, 865-874.

4. Boosalis, M. S., Petruska, J. \& Goodman, M. F. (1987) J. Biol. Chem. 262, 14689-14696.

5. Goodman, M. F., Creighton, S., Bloom, L. B. \& Petruska, J. (1993) Crit. Rev. Biochem. Mol. Biol. 28, 83-126.

6. Parker, W. B., Bapat, A. R., Shen, J. X., Townsend, A. J. \& Cheng, Y. C. (1988) Mol. Pharmacol. 34, 485-491.

7. Hentosh, P., Koob, R. \& Blakley, R. L. (1990) J. Biol. Chem. 265, 4033-4040.

8. Chunduru, S. K., Appleman, J. R. \& Blakley, R. L. (1993) Arch. Biochem. Biophys. 302, 19-30.

9. Hentosh, P., McCastlain, J. C. \& Blakley, R. L. (1991) Biochemistry 30, 547-554.

10. Henthosh, P. \& Grippo, P. (1994) Mol. Pharmacol. 45, 955-961.

11. Goodman, M.F. (1988) Mutation Res. 200, 11-20. 
12. Petruska, J., Goodman, M.F., Boosalis, M.S., Sowers, L.C., Cheong, C. \& Tinoco, I. (1988) Proc. Natl. Acad. Sci. U.S.A. 85, 6252-6256.

13. Singer, B. \& Dosanjh, M.K. (1990) Mutation Res. 233, 45-51.
14. Dosanjh, M. K., Galeros, G., Goodman, M. F. \& Singer, B. (1991) Biochemistry 30, 1159511599.

15. Dosanjh, M. K., Menichini, P., Eritja, R. \& Singer, B., (1993) Carcinogenesis 14, 19151919.

16. Henthosh, P. \& Tibudan, M. (1995) Mol. Pharmacol. 48, 897-904. 